\title{
Computational Investigation of Mechanisms for Ring-Opening Polymerization of \&-Caprolactone: Evidence for Bifunctional Catalysis by Alcohols.
}

\author{
Nicholas Buis, ${ }^{\dagger}$ Samuel A. French, ${ }^{\dagger}$ Giuseppe D Ruggiero, ${ }^{\dagger}$ Bruno Stengel, Arran A. D. \\ Tulloch and Ian H Williams ${ }^{\dagger *}$ \\ ${ }^{\dagger}$ Department of Chemistry, University of Bath, Bath, BA2 7AY, UK, ${ }^{\star}$ Johnson Matthey \\ Technology Centre, Blounts Court, Sonning Common, Reading, RG4 9NH, UK, and ${ }^{\S}$ Johnson \\ Matthey Catalysts, P.O. Box 1, Belasis Avenue, Billingham, Cleveland, TS23 1LB, UK
}

\section{Supporting Information}

Full citation for Gaussian03

Table S1. B3LYP/6-31G* total energies, zero-point vibrational energies, and transition frequencies in vacuum for methanolysis of $\varepsilon$-caprolactone.

Cartesian coordinates (Ångström) for B3LYP/6-31G* optimised structures in vacuum:

4 reactant complex for methanolysis of $\varepsilon-\mathrm{CL}$ with one $\mathrm{CH} 3 \mathrm{OH} \quad \mathrm{S} 4$

TS1 for addition step of stepwise mechanism for methanolysis of $\varepsilon$-CL with one $\mathrm{CH} 3 \mathrm{OH} \quad$ S5

5a tetrahedral adduct intermediate for methanolysis of $\varepsilon$-CL with one $\mathrm{CH} 3 \mathrm{OH} \quad \mathrm{S} 6$

TS $5 \mathbf{a} \rightarrow \mathbf{5 b}$ for conformational isomerism of tetrahedral adduct intermediate

$\begin{array}{ll}\text { for methanolysis of } \varepsilon-\mathrm{CL} \text { with one } \mathrm{CH} 3 \mathrm{OH} & \mathrm{S} 7\end{array}$

5b tetrahedral adduct intermediate for methanolysis of $\varepsilon-\mathrm{CL}$ with one $\mathrm{CH} 3 \mathrm{OH} \quad \mathrm{S} 8$

TS2 for elimination step of stepwise mechanism for methanolysis of $\varepsilon$-CL with one $\mathrm{CH} 3 \mathrm{OH} \quad \mathrm{S} 9$

6 product complex for methanolysis of $\varepsilon$-CL with one $\mathrm{CH} 3 \mathrm{OH} \quad \mathrm{S} 10$

TS3 for concerted mechanism for methanolysis of $\varepsilon-\mathrm{CL}$ with one $\mathrm{CH} 3 \mathrm{OH} \quad \mathrm{S} 11$

8 reactant complex for methanolysis of $\varepsilon-\mathrm{CL}$ with two $\mathrm{CH} 3 \mathrm{OH} \quad \mathrm{S} 12$

TS4 for addition step of stepwise mechanism for methanolysis of $\varepsilon$-CL with two CH3OH $\quad$ S13

9a tetrahedral adduct intermediate for methanolysis of $\varepsilon$-CL with two $\mathrm{CH} 3 \mathrm{OH} \quad \mathrm{S} 14$

9b tetrahedral adduct intermediate for methanolysis of $\varepsilon-\mathrm{CL}$ with two $\mathrm{CH} 3 \mathrm{OH} \quad \mathrm{S} 15$

TS5 for elimination step of stepwise mechanism for methanolysis of $\varepsilon-\mathrm{CL}$ with two $\mathrm{CH} 3 \mathrm{OH}$ S16

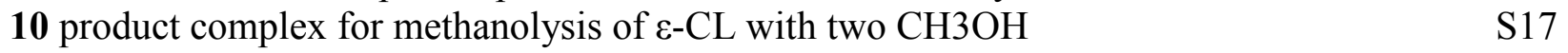

TS6 for concerted mechanism for methanolysis of $\varepsilon-\mathrm{CL}$ with two $\mathrm{CH} 3 \mathrm{OH} \quad \mathrm{S} 18$

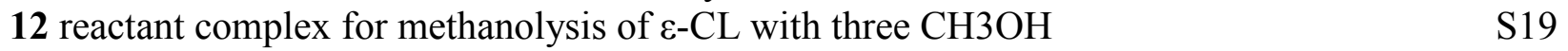

TS7 for addition step of stepwise mechanism for methanolysis of $\varepsilon-\mathrm{CL}$ with three $\mathrm{CH} 3 \mathrm{OH} \quad$ S20

13a tetrahedral adduct intermediate for methanolysis of $\varepsilon$-CL with three $\mathrm{CH} 3 \mathrm{OH} \quad \mathrm{S} 21$

13b tetrahedral adduct intermediate for methanolysis of $\varepsilon-\mathrm{CL}$ with three $\mathrm{CH} 3 \mathrm{OH} \quad \mathrm{S} 22$

TS8 for elimination step of stepwise mechanism for methanolysis of $\varepsilon-\mathrm{CL}$

$\begin{array}{ll}\text { with three } \mathrm{CH} 3 \mathrm{OH} & \text { S23 }\end{array}$

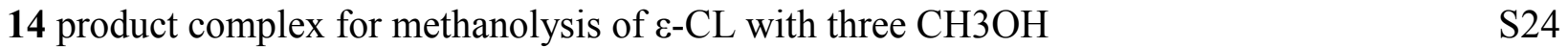

TS9 for concerted mechanism for methanolysis of $\varepsilon-\mathrm{CL}$ with three $\mathrm{CH} 3 \mathrm{OH} \quad \mathrm{S} 25$ 


\section{Full citation for Gaussian03}

Gaussian 03, Revision C.02, Frisch, M. J.; Trucks, G. W.; Schlegel, H. B.; Scuseria, G. E.; Robb, M. A.; Cheeseman, J. R.; Montgomery, Jr., J. A.; Vreven, T.; Kudin, K. N.; Burant, J. C.; Millam, J. M.; Iyengar, S. S.; Tomasi, J.; Barone, V.; Mennucci, B.; Cossi, M.; Scalmani, G.; Rega, N.; Petersson, G. A.; Nakatsuji, H.; Hada, M.; Ehara, M.; Toyota, K.; Fukuda, R.; Hasegawa, J.; Ishida, M.; Nakajima, T.; Honda, Y.; Kitao, O.; Nakai, H.; Klene, M.; Li, X.; Knox, J. E.; Hratchian, H. P.; Cross, J. B.; Bakken, V.; Adamo, C.; Jaramillo, J.; Gomperts, R.; Stratmann, R. E.; Yazyev, O.; Austin, A. J.; Cammi, R.; Pomelli, C.; Ochterski, J. W.; Ayala, P. Y.; Morokuma, K.; Voth, G. A.; Salvador, P.; Dannenberg, J. J.; Zakrzewski, V. G.; Dapprich, S.; Daniels, A. D.; Strain, M. C.; Farkas, O.; Malick, D. K.; Rabuck, A. D.; Raghavachari, K.; Foresman, J. B.; Ortiz, J. V.; Cui, Q.; Baboul, A. G.; Clifford, S.; Cioslowski, J.; Stefanov, B. B.; Liu, G.; Liashenko, A.; Piskorz, P.; Komaromi, I.; Martin, R. L.; Fox, D. J.; Keith, T.; AlLaham, M. A.; Peng, C. Y.; Nanayakkara, A.; Challacombe, M.; Gill, P. M. W.; Johnson, B.; Chen, W.; Wong, M. W.; Gonzalez, C.; and Pople, J. A.; Gaussian, Inc., Wallingford CT, 2004. 
Table S1. B3LYP/6-31G* total energies $\left(E_{\text {tot }} /\right.$ Hartree), zero-point vibrational energies $\left(E_{\mathrm{zp}} / \mathrm{kJ} \mathrm{mol}^{-1}\right)$, and transition frequencies $\left(v^{\ddagger} / \mathrm{cm}^{-1}\right)$ in vacuum for methanolysis of $\varepsilon$ caprolactone.

\begin{tabular}{|c|c|c|c|}
\hline species & $E_{\mathrm{tot}}$ & $E_{\mathrm{zp}}$ & $v^{\ddagger}$ \\
\hline$\varepsilon-C L(1)$ & -385.11587 & 413.3 & \\
\hline $\mathrm{CH}_{3} \mathrm{OH}(2)$ & -115.71441 & 135.1 & \\
\hline$\varepsilon-\mathrm{CL}+\mathrm{CH}_{3} \mathrm{OH}(\mathbf{3})$ & -500.83027 & 548.4 & \\
\hline$\varepsilon-\mathrm{CL} . \mathrm{CH}_{3} \mathrm{OH}(\mathbf{4})$ & -500.84214 & 553.0 & \\
\hline addition TS (TS1) & -500.77638 & 543.1 & $1612 i$ \\
\hline intermediate (5a) & -500.83157 & 558.5 & \\
\hline conformational TS $(\mathbf{5 a} \rightarrow \mathbf{5 b})$ & -500.82596 & 556.8 & $115 i$ \\
\hline intermediate (5b) & -500.83530 & 558.2 & \\
\hline elimination TS (TS2) & -500.78297 & 542.5 & $1513 i$ \\
\hline product (6) & -500.84612 & 555.6 & \\
\hline concerted TS (TS3) & -500.77675 & 541.4 & $1154 i$ \\
\hline$\varepsilon-\mathrm{CL}+2 \mathrm{CH}_{3} \mathrm{OH}(7)$ & -616.54468 & 683.6 & \\
\hline$\varepsilon-\mathrm{CL} .2 \mathrm{CH}_{3} \mathrm{OH}(\mathbf{8})$ & -616.57916 & 696.7 & \\
\hline addition TS (TS4) & -616.52647 & 681.9 & $1399 i$ \\
\hline intermediate $+\mathrm{CH}_{3} \mathrm{OH}$ (9a) & -616.56315 & 702.6 & \\
\hline intermediate $+\mathrm{CH}_{3} \mathrm{OH}(\mathbf{9 b})$ & -616.56785 & 701.9 & \\
\hline elimination TS (TS5) & -616.53388 & 682.0 & $1433 i$ \\
\hline product. $\mathrm{CH}_{3} \mathrm{OH}(\mathbf{1 0})$ & -616.58432 & 696.3 & \\
\hline concerted TS (TS6) & -616.51118 & 678.4 & $1320 i$ \\
\hline$\varepsilon-\mathrm{CL}+3 \mathrm{CH}_{3} \mathrm{OH}(\mathbf{1 1})$ & -732.25908 & 818.7 & \\
\hline$\varepsilon-\mathrm{CL} .3 \mathrm{CH}_{3} \mathrm{OH}(\mathbf{1 2})$ & -732.31480 & 838.8 & \\
\hline addition TS (TS7) & -732.26732 & 814.9 & $1882 i$ \\
\hline intermediate $+2 \mathrm{CH}_{3} \mathrm{OH}$ (13a) & -732.30220 & 843.6 & \\
\hline intermediate $+2 \mathrm{CH}_{3} \mathrm{OH}$ (13b) & -732.30238 & 843.3 & \\
\hline elimination TS (TS8) & -732.26568 & 819.3 & $1185 i$ \\
\hline product. $2 \mathrm{CH}_{3} \mathrm{OH}$ (14) & -732.31831 & 838.0 & \\
\hline concerted TS (TS9) & -732.24856 & 825.5 & $469 i$ \\
\hline \multicolumn{4}{|c|}{ single-point energies for $\varepsilon$-CL. $\mathrm{CH}_{3} \mathrm{OH}$ fragments: } \\
\hline 4 as in 8 & -500.83759 & & \\
\hline 4 as in TS4 & -500.74772 & & \\
\hline 4 as in TS5 & -500.76335 & & \\
\hline 4 as in TS6 & -500.73357 & & \\
\hline $\mathbf{4}$ as in $\mathbf{1 2}$ & -500.83671 & & \\
\hline 4 as in TS7 & -500.76491 & & \\
\hline 4 as in TS8 & -500.74205 & & \\
\hline 4 as in TS9 & -500.66912 & & \\
\hline
\end{tabular}




\section{Cartesian coordinates (Ångström) for B3LYP/6-31G* optimised structures}

\begin{tabular}{|c|c|c|c|}
\hline \multicolumn{4}{|c|}{ in vacuum } \\
\hline \multicolumn{4}{|c|}{4 reactant complex for methanolysis of $\varepsilon-\mathrm{C}$} \\
\hline \multicolumn{4}{|c|}{ Total energy $=-500.631487$} \\
\hline 6 & -4.547674 & -0.149487 & 0.287141 \\
\hline 8 & -3.477947 & 0.768195 & 0.332330 \\
\hline 8 & -1.194948 & -0.971014 & -0.236488 \\
\hline 6 & -0.082463 & -0.514007 & -0.413426 \\
\hline 6 & 1.137036 & -1.401060 & -0.593502 \\
\hline 6 & 2.156226 & -1.296748 & 0.563301 \\
\hline 6 & 3.062097 & -0.060384 & 0.497953 \\
\hline 6 & 2.322199 & 1.284024 & 0.510078 \\
\hline 6 & 1.331658 & 1.475588 & -0.637146 \\
\hline 8 & 0.061020 & 0.826839 & -0.420079 \\
\hline 1 & 3.059175 & 2.095585 & 0.449452 \\
\hline 1 & 1.781000 & 1.424325 & 1.454959 \\
\hline 1 & 3.672854 & -0.118359 & -0.415225 \\
\hline 1 & 3.766132 & -0.088963 & 1.338811 \\
\hline 1 & 2.785173 & -2.194439 & 0.537301 \\
\hline 1 & 1.618981 & -1.325710 & 1.520455 \\
\hline 1 & 1.631818 & -1.171219 & -1.547219 \\
\hline 1 & 0.747065 & -2.418503 & -0.664152 \\
\hline 1 & -2.664481 & 0.272219 & 0.122741 \\
\hline 1 & -4.450151 & -0.961853 & 1.027271 \\
\hline 1 & -5.465157 & 0.403485 & 0.516740 \\
\hline 1 & -4.675043 & -0.614807 & -0.705180 \\
\hline 1 & 1.756098 & 1.150509 & -1.595754 \\
\hline 1 & 1.057058 & 2.528005 & -0.732702 \\
\hline
\end{tabular}


TS1 for addition step of stepwise mechanism for methanolysis of $\varepsilon$ - $\mathrm{CL}$ with one $\mathrm{CH} 3 \mathrm{OH}$

Total energy $=-500.569512 ;$ transition frequency $=1612 i \mathrm{~cm}^{-1}$

$\begin{array}{rrrr}6 & -0.575810 & -0.444980 & 0.363111 \\ 8 & -2.210048 & -0.378800 & -0.568987 \\ 8 & -1.217764 & -1.122736 & 1.279796 \\ 1 & -2.150705 & -0.997519 & 0.562483 \\ 6 & -2.889199 & 0.858158 & -0.550230 \\ 1 & -2.744774 & 1.400650 & 0.396434 \\ 1 & -2.542327 & 1.497617 & -1.374781 \\ 1 & -3.964698 & 0.684638 & -0.689597 \\ 6 & 0.277363 & -1.180498 & -0.654185 \\ 1 & -0.207717 & -2.147945 & -0.796067 \\ 1 & 0.261794 & -0.666753 & -1.620994 \\ 6 & 1.732518 & -1.359840 & -0.170028 \\ 1 & 1.733640 & -1.616873 & 0.897324 \\ 1 & 2.165913 & -2.219656 & -0.694782 \\ 6 & 2.616936 & -0.131215 & -0.433742 \\ 1 & 2.650672 & 0.045582 & -1.519096 \\ 1 & 3.647354 & -0.352811 & -0.128348 \\ 6 & 2.163126 & 1.160770 & 0.262716 \\ 1 & 2.275238 & 1.071218 & 1.351053 \\ 1 & 2.816094 & 1.985682 & -0.052870 \\ 6 & 0.719384 & 1.574124 & -0.019983 \\ 1 & 0.474705 & 1.522626 & -1.087328 \\ 1 & 0.541902 & 2.597545 & 0.318562 \\ 8 & -0.225063 & 0.793647 & 0.745699\end{array}$


5a tetrahedral adduct intermediate for methanolysis of $\varepsilon$-CL with one $\mathrm{CH} 3 \mathrm{OH}$

Total energy $+\mathrm{ZPE}=-500.618801$

$\begin{array}{rrrr}6 & -2.859330 & 0.725089 & -0.313537 \\ 8 & -1.788653 & -0.067313 & -0.826883 \\ 6 & -0.765804 & -0.371779 & 0.099725 \\ 8 & -0.240767 & 0.775518 & 0.700559 \\ 6 & 0.680423 & 1.564004 & -0.057709 \\ 6 & 2.125826 & 1.175565 & 0.256365 \\ 6 & 2.566098 & -0.180398 & -0.314535 \\ 6 & 1.621372 & -1.355785 & -0.009009 \\ 6 & 0.247869 & -1.224313 & -0.685866 \\ 8 & -1.276681 & -1.071588 & 1.212176 \\ 1 & -1.764813 & -1.828936 & 0.851676 \\ 1 & -3.351180 & 0.234487 & 0.533835 \\ 1 & -2.506877 & 1.710603 & 0.008980 \\ 1 & -3.569382 & 0.838917 & -1.135922 \\ 1 & -0.210756 & -2.211563 & -0.820554 \\ 1 & 0.357120 & -0.807864 & -1.693746 \\ 1 & 1.483462 & -1.469766 & 1.073448 \\ 1 & 2.097416 & -2.278645 & -0.363393 \\ 1 & 2.668959 & -0.094851 & -1.406854 \\ 1 & 3.568496 & -0.413635 & 0.067628 \\ 1 & 2.230334 & 1.173837 & 1.349084 \\ 1 & 2.791306 & 1.963233 & -0.124308 \\ 1 & 0.458701 & 1.498466 & -1.131140 \\ 1 & 0.497307 & 2.598486 & 0.251850\end{array}$


TS 5a $\rightarrow \mathbf{5 b}$ for conformational isomerism of tetrahedral adduct intermediate for methanolysis of $\varepsilon$-CL with one $\mathrm{CH} 3 \mathrm{OH}$

Total energy $+\mathrm{ZPE}=-500.613848$; transition frequency $=115 i \mathrm{~cm}^{-1}$

$\begin{array}{rrrr}6 & -2.622201 & 0.928354 & -0.593440 \\ 8 & -2.034284 & -0.356802 & -0.471618 \\ 6 & -0.773549 & -0.486802 & 0.185262 \\ 8 & -0.929411 & -1.383150 & 1.244861 \\ 8 & -0.375227 & 0.743225 & 0.747412 \\ 6 & 0.573963 & 1.586358 & 0.087844 \\ 6 & 2.021902 & 1.177587 & 0.366129 \\ 6 & 2.508715 & -0.042151 & -0.427871 \\ 6 & 1.641698 & -1.301598 & -0.278140 \\ 6 & 0.204835 & -1.134702 & -0.799026 \\ 1 & -1.535228 & -0.961491 & 1.877185 \\ 1 & -2.787283 & 1.413782 & 0.375434 \\ 1 & -2.031613 & 1.607231 & -1.224324 \\ 1 & -3.587848 & 0.759106 & -1.077773 \\ 1 & -0.234621 & -2.109518 & -1.030807 \\ 1 & 0.192367 & -0.561362 & -1.733776 \\ 1 & 1.606630 & -1.621462 & 0.770062 \\ 1 & 2.120677 & -2.116046 & -0.836198 \\ 1 & 2.557675 & 0.225826 & -1.494333 \\ 1 & 3.538420 & -0.273570 & -0.125321 \\ 1 & 2.111841 & 0.993235 & 1.444617 \\ 1 & 2.668262 & 2.036602 & 0.136711 \\ 1 & 0.377395 & 1.636960 & -0.991997 \\ 1 & 0.382526 & 2.582246 & 0.500734\end{array}$


$\mathbf{5 b}$ tetrahedral adduct intermediate for methanolysis of $\varepsilon$-CL with one $\mathrm{CH} 3 \mathrm{OH}$

Total energy $+\mathrm{ZPE}=-500.622670$

$\begin{array}{rrrr}6 & -2.890561 & 0.638929 & -0.356964 \\ 8 & -1.732193 & -0.011127 & -0.867150 \\ 6 & -0.769929 & -0.379258 & 0.091373 \\ 8 & -1.331472 & -1.105753 & 1.152649 \\ 6 & 0.232475 & -1.271453 & -0.650300 \\ 6 & 1.616004 & -1.363615 & 0.010883 \\ 6 & 2.549418 & -0.191184 & -0.342424 \\ 6 & 2.135666 & 1.175233 & 0.225330 \\ 6 & 0.685245 & 1.570752 & -0.049966 \\ 8 & -0.215929 & 0.765031 & 0.717565 \\ 1 & -1.394249 & -0.487696 & 1.899332 \\ 1 & -3.496445 & 0.904732 & -1.226083 \\ 1 & -2.630726 & 1.551324 & 0.194681 \\ 1 & -3.464441 & -0.028982 & 0.29551 \\ 1 & 0.432317 & 1.508727 & -1.115829 \\ 1 & 0.508196 & 2.602880 & 0.271534 \\ 1 & 1.497062 & -1.449069 & 1.098509 \\ 1 & 2.276066 & 1.184885 & 1.314415 \\ 1 & 2.794860 & 1.953859 & -0.183909 \\ 1 & 2.095127 & -2.292709 & -0.322103 \\ 1 & 2.614069 & -0.120181 & -1.438609 \\ 1 & 3.564671 & -0.420039 & 0.007289 \\ 1 & 0.326302 & -0.910235 & -1.680549 \\ 1 & -0.235971 & -2.259131 & -0.706340\end{array}$


TS2 for elimination step of stepwise mechanism for methanolysis of $\varepsilon$-CL with one $\mathrm{CH} 3 \mathrm{OH}$ Total energy $+\mathrm{ZPE}=-500.576287$; transition frequency $=1513 i \mathrm{~cm}^{-1}$

$\begin{array}{rrrr}6 & -0.943135 & -0.457725 & 0.084875 \\ 8 & -0.148610 & 1.254172 & 0.100288 \\ 8 & -1.036673 & -0.292358 & 1.383498 \\ 1 & -0.439646 & 0.706143 & 1.231763 \\ 6 & 1.102223 & 1.669559 & -0.399132 \\ 1 & 1.100003 & 1.553064 & -1.495287 \\ 1 & 1.200117 & 2.748635 & -0.201013 \\ 6 & 2.307056 & 0.938657 & 0.198949 \\ 1 & 3.211135 & 1.497286 & -0.083233 \\ 1 & 2.241616 & 0.993595 & 1.295453 \\ 6 & 2.486909 & -0.521430 & -0.241951 \\ 1 & 2.579884 & -0.555824 & -1.338243 \\ 1 & 3.448902 & -0.876951 & 0.151121 \\ 6 & 1.406001 & -1.523682 & 0.199786 \\ 1 & 1.797377 & -2.531997 & 0.016537 \\ 1 & 1.238323 & -1.450899 & 1.281259 \\ 6 & 0.051442 & -1.424390 & -0.517090 \\ 1 & 0.168137 & -1.199377 & -1.583117 \\ 1 & -0.455218 & -2.398925 & -0.465103 \\ 8 & -2.033405 & -0.357059 & -0.680376 \\ 6 & -3.112101 & 0.445732 & -0.167988 \\ 1 & -3.911084 & 0.358196 & -0.904986 \\ 1 & -2.781514 & 1.483523 & -0.082478 \\ 1 & -3.438914 & 0.075171 & 0.805358\end{array}$


6 product complex for methanolysis of $\varepsilon$ - $\mathrm{CL}$ with one $\mathrm{CH} 3 \mathrm{OH}$

Total energy $+\mathrm{ZPE}=-500.634453$

$\begin{array}{rrrr}6 & 2.723529 & -1.274567 & -0.225973 \\ 8 & 2.214864 & 0.039336 & -0.524171 \\ 6 & 1.252137 & 0.499878 & 0.292551 \\ 6 & 0.748865 & 1.859169 & -0.153688 \\ 6 & -0.765914 & 2.040706 & 0.048842 \\ 6 & -1.621249 & 0.941003 & -0.635084 \\ 6 & -2.272603 & -0.067298 & 0.343572 \\ 6 & -2.170198 & -1.524389 & -0.116447 \\ 8 & -0.821262 & -1.961247 & -0.253369 \\ 8 & 0.851250 & -0.112872 & 1.267229 \\ 1 & -0.338661 & -1.658429 & 0.535698 \\ 1 & -1.789044 & 0.005881 & 1.325778 \\ 1 & -2.720464 & -2.170144 & 0.587920 \\ 1 & -3.330339 & 0.180469 & 0.505583 \\ 1 & -2.629468 & -1.656714 & -1.103672 \\ 1 & -2.403132 & 1.409963 & -1.243799 \\ 1 & -0.988943 & 0.382635 & -1.336657 \\ 1 & 1.022386 & 1.997471 & -1.204377 \\ 1 & -1.026744 & 3.030468 & -0.343940 \\ 1 & 1.300472 & 2.618041 & 0.419007 \\ 1 & -0.983324 & 2.069456 & 1.122529 \\ 1 & 3.058076 & -1.333387 & 0.812427 \\ 1 & 3.558543 & -1.424444 & -0.910675 \\ 1 & 1.944434 & -2.020021 & -0.405971\end{array}$


TS3 for concerted mechanism for methanolysis of $\varepsilon$ - $\mathrm{CL}$ with one $\mathrm{CH} 3 \mathrm{OH}$

Total energy $+\mathrm{ZPE}=-500.570492 ;$ transition frequency $=1154 i \mathrm{~cm}^{-1}$

$\begin{array}{rrrr}8 & -1.936370 & 0.234841 & -0.754541 \\ 6 & -0.716641 & -0.562314 & 0.305447 \\ 8 & -0.233545 & 1.174182 & 0.384181 \\ 1 & -1.215421 & 1.095749 & -0.328284 \\ 8 & -1.140662 & -0.940972 & 1.349548 \\ 6 & -3.244283 & 0.234212 & -0.193683 \\ 1 & -3.219860 & -0.183150 & 0.821388 \\ 1 & -3.903051 & -0.375409 & -0.821756 \\ 1 & -3.636585 & 1.256807 & -0.150269 \\ 6 & 0.970763 & 1.682408 & -0.183016 \\ 1 & 0.956621 & 1.610859 & -1.283280 \\ 1 & 1.000071 & 2.750442 & 0.070731 \\ 6 & 2.219261 & 0.993362 & 0.375919 \\ 1 & 3.067266 & 1.678579 & 0.240983 \\ 1 & 2.084441 & 0.872288 & 1.458845 \\ 6 & 2.587104 & -0.347097 & -0.280739 \\ 1 & 2.734078 & -0.180112 & -1.358546 \\ 1 & 3.561989 & -0.668117 & 0.109405 \\ 6 & 1.596263 & -1.505661 & -0.093804 \\ 1 & 2.012284 & -2.391640 & -0.588985 \\ 1 & 1.501693 & -1.761714 & 0.968786 \\ 1 & -0.308062 & -2.206018 & -0.889587 \\ 6 & 0.190268 & -1.253011 & -0.675113 \\ 1 & 0.232745 & -0.694378 & -1.613004\end{array}$


8 reactant complex for methanolysis of $\varepsilon-\mathrm{CL}$ with two $\mathrm{CH} 3 \mathrm{OH}$

Total energy $+\mathrm{ZPE}=-616.313746$

$\begin{array}{rrrr}6 & 2.118914 & 2.675615 & -0.459574 \\ 8 & 1.439906 & 1.466413 & -0.742767 \\ 8 & 2.906689 & -0.627897 & 0.372404 \\ 6 & 3.131537 & -1.806499 & -0.391405 \\ 8 & 0.524635 & -0.653056 & 1.825344 \\ 6 & -0.281908 & -0.337231 & 0.966387 \\ 6 & -1.312382 & 0.751416 & 1.187080 \\ 6 & -2.782280 & 0.320465 & 1.003772 \\ 6 & -3.240841 & 0.232536 & -0.456979 \\ 6 & -2.474542 & -0.789531 & -1.305180 \\ 6 & -0.967795 & -0.557958 & -1.375391 \\ 8 & -0.257067 & -1.014250 & -0.197858 \\ 1 & -2.859865 & -0.761458 & -2.333374 \\ 1 & -2.650498 & -1.808405 & -0.934551 \\ 1 & -3.139027 & 1.225235 & -0.919479 \\ 1 & -4.310725 & -0.010480 & -0.483200 \\ 1 & -3.410403 & 1.053628 & 1.523949 \\ 1 & -2.949743 & -0.639346 & 1.511279 \\ 1 & -1.067770 & 1.582528 & 0.513003 \\ 1 & -1.143321 & 1.103037 & 2.207367 \\ 1 & 2.021195 & 0.725295 & -0.454186 \\ 1 & 3.056683 & 2.773060 & -1.028677 \\ 1 & 1.462009 & 3.502662 & -0.749432 \\ 1 & 2.357132 & 2.786184 & 0.609734 \\ 1 & -0.700291 & 0.486254 & -1.562027 \\ 1 & -0.531463 & -1.163186 & -2.173282 \\ 1 & 3.559089 & -2.609960 & 0.225189 \\ 1 & 2.209536 & -2.175132 & -0.860135 \\ 1 & 3.851952 & -1.555393 & -1.175178 \\ 1 & 2.187978 & -0.817091 & 1.013753\end{array}$


TS4 for addition step of stepwise mechanism for methanolysis of $\varepsilon-\mathrm{CL}$ with two $\mathrm{CH} 3 \mathrm{OH}$

Total energy $+\mathrm{ZPE}=-616.266704 ;$ transition frequency $=1399 i \mathrm{~cm}^{-1}$

$\begin{array}{rrrr}6 & 1.288375 & 1.217359 & 1.839566 \\ 8 & 1.311673 & -0.082930 & 1.288768 \\ 6 & 0.058049 & -0.523607 & 0.046764 \\ 8 & -0.105079 & 0.662869 & -0.640294 \\ 6 & -1.223472 & 1.530600 & -0.390105 \\ 6 & -2.530395 & 0.995180 & -0.979996 \\ 6 & -3.219492 & -0.092239 & -0.142539 \\ 6 & -2.354467 & -1.323977 & 0.162924 \\ 6 & -1.068045 & -1.000033 & 0.949320 \\ 8 & 0.665394 & -1.432103 & -0.630179 \\ 8 & 2.913514 & -0.708705 & -0.374236 \\ 6 & 3.340938 & 0.337735 & -1.248542 \\ 1 & 2.194770 & 1.367844 & 2.440358 \\ 1 & 0.422624 & 1.335369 & 2.506044 \\ 1 & 1.247207 & 2.001144 & 1.069210 \\ 1 & -1.279330 & -0.269426 & 1.738181 \\ 1 & -0.679718 & -1.897199 & 1.435217 \\ 1 & -2.952363 & -2.029702 & 0.752641 \\ 1 & -2.083393 & -1.845111 & -0.763804 \\ 1 & -4.133781 & -0.410845 & -0.659629 \\ 1 & -3.546679 & 0.351820 & 0.809731 \\ 1 & -3.218840 & 1.843664 & -1.095862 \\ 1 & -2.313243 & 0.624871 & -1.990244 \\ 1 & -1.327073 & 1.742262 & 0.681421 \\ 1 & -0.942848 & 2.464368 & -0.885648 \\ 1 & 2.314054 & -0.300850 & 0.546142 \\ 1 & 4.125306 & 0.922290 & -0.755948 \\ 1 & 3.757730 & -0.107719 & -2.157383 \\ 1 & 2.509185 & 0.999120 & -1.522527 \\ 1 & 1.873442 & -1.151056 & -0.724715\end{array}$


9a tetrahedral adduct intermediate for methanolysis of $\varepsilon$-CL with two $\mathrm{CH} 3 \mathrm{OH}$

Total energy $+\mathrm{ZPE}=-616.295511$

$\begin{array}{rrrr}6 & -1.197404 & 2.159079 & 1.144016 \\ 8 & -1.163109 & 1.379405 & -0.047915 \\ 6 & -0.034839 & 0.506749 & -0.219638 \\ 6 & 1.272808 & 1.308206 & -0.339977 \\ 6 & 2.432113 & 0.553918 & -1.009085 \\ 6 & 3.045825 & -0.569976 & -0.163944 \\ 6 & 2.039428 & -1.598874 & 0.373423 \\ 6 & 1.054357 & -1.028009 & 1.400563 \\ 8 & -0.112175 & -0.376479 & 0.889641 \\ 8 & -0.243798 & -0.158115 & -1.408171 \\ 8 & -2.941466 & -0.599795 & -0.952823 \\ 6 & -3.306493 & -1.491096 & 0.098204 \\ 1 & -2.175988 & 2.645245 & 1.162394 \\ 1 & -0.422120 & 2.935779 & 1.137871 \\ 1 & -1.080583 & 1.531890 & 2.033579 \\ 1 & 1.569133 & 1.673862 & 0.650987 \\ 1 & 1.024843 & 2.185413 & -0.947027 \\ 1 & 3.218623 & 1.280701 & -1.250291 \\ 1 & 2.071676 & 0.149611 & -1.959867 \\ 1 & 3.806865 & -1.087298 & -0.762614 \\ 1 & 3.579466 & -0.131128 & 0.693039 \\ 1 & 2.596940 & -2.401337 & 0.875620 \\ 1 & 1.476650 & -2.062882 & -0.444596 \\ 1 & 1.589570 & -0.347090 & 2.082159 \\ 1 & 0.644882 & -1.838316 & 2.011934 \\ 1 & -2.678754 & 0.251281 & -0.547748 \\ 1 & -4.226065 & -1.162977 & 0.603638 \\ 1 & -3.497200 & -2.465686 & -0.359260 \\ 1 & -2.504565 & -1.594391 & 0.839956 \\ 1 & -1.143761 & -0.562801 & -1.366998\end{array}$


9b tetrahedral adduct intermediate for methanolysis of $\varepsilon$-CL with two $\mathrm{CH} 3 \mathrm{OH}$

Total energy $+\mathrm{ZPE}=-616.300453$

$\begin{array}{rrrr}6 & -1.504236 & 2.359927 & 0.750358 \\ 8 & -0.192835 & 2.094262 & 0.264725 \\ 6 & -0.013888 & 0.852412 & -0.376052 \\ 8 & -0.991172 & 0.564000 & -1.312842 \\ 8 & -0.091251 & -0.207573 & 0.600067 \\ 6 & 0.972022 & -0.298491 & 1.558554 \\ 6 & 2.010280 & -1.327006 & 1.111929 \\ 6 & 2.844121 & -0.909031 & -0.108683 \\ 6 & 2.038700 & -0.408709 & -1.322019 \\ 6 & 1.342642 & 0.940556 & -1.086297 \\ 8 & -2.698725 & -1.005215 & 0.233723 \\ 6 & -2.815144 & -2.260669 & -0.431091 \\ 1 & -1.760986 & 0.145342 & -0.862009 \\ 1 & -1.448388 & 3.325662 & 1.257502 \\ 1 & -1.836859 & 1.596988 & 1.465466 \\ 1 & -2.226873 & 2.422236 & -0.071055 \\ 1 & 1.408376 & 0.693526 & 1.722740 \\ 1 & 0.506724 & -0.612963 & 2.499446 \\ 1 & 1.296468 & -1.155888 & -1.630088 \\ 1 & 1.475438 & -2.261759 & 0.895566 \\ 1 & 2.680774 & -1.537938 & 1.956976 \\ 1 & 2.731708 & -0.294896 & -2.164958 \\ 1 & 3.545061 & -0.115495 & 0.190211 \\ 1 & 3.464400 & -1.762217 & -0.413344 \\ 1 & 1.994575 & 1.608758 & -0.512935 \\ 1 & 1.138825 & 1.441287 & -2.037771 \\ 1 & -1.829256 & -0.981931 & 0.681907 \\ 1 & -3.755684 & -2.244865 & -0.987505 \\ 1 & -1.991004 & -2.428101 & -1.138346 \\ 1 & -2.848417 & -3.095474 & 0.282614\end{array}$


TS5 for elimination step of stepwise mechanism for methanolysis of $\varepsilon$-CL with two $\mathrm{CH} 3 \mathrm{OH}$ Total energy $+\mathrm{ZPE}=-616.274057$; transition frequency $=1432 i \mathrm{~cm}^{-1}$

$\begin{array}{rrrr}6 & 0.640225 & -0.886790 & -0.410731 \\ 8 & 0.117030 & 0.219213 & 0.928421 \\ 8 & 1.382967 & -0.083459 & -1.108220 \\ 1 & 1.001451 & 1.058992 & -1.017823 \\ 6 & -1.070963 & -0.028245 & 1.668502 \\ 1 & -1.166218 & -1.112657 & 1.819557 \\ 1 & -0.937769 & 0.419846 & 2.663769 \\ 6 & -2.322360 & 0.551282 & 1.004587 \\ 1 & -3.138633 & 0.546935 & 1.740693 \\ 1 & -2.125225 & 1.606208 & 0.768829 \\ 6 & -2.793771 & -0.183290 & -0.259524 \\ 1 & -3.193494 & -1.167725 & 0.026540 \\ 1 & -3.639507 & 0.373499 & -0.684258 \\ 6 & -1.740490 & -0.392561 & -1.363094 \\ 1 & -2.266379 & -0.749803 & -2.256939 \\ 1 & -1.280590 & 0.562021 & -1.645494 \\ 6 & -0.652260 & -1.424490 & -1.014360 \\ 1 & -1.054333 & -2.205029 & -0.360016 \\ 1 & -0.315691 & -1.931145 & -1.927748 \\ 8 & 1.260964 & -1.882324 & 0.267673 \\ 6 & 2.593164 & -1.604216 & 0.713818 \\ 1 & 2.918292 & -2.504015 & 1.239060 \\ 1 & 2.590040 & -0.750454 & 1.397865 \\ 1 & 3.252917 & -1.396497 & -0.131858 \\ 1 & 0.215012 & 1.308124 & 0.354947 \\ 8 & 0.528544 & 2.060198 & -0.514870 \\ 6 & 1.565442 & 2.946075 & -0.102288 \\ 1 & 1.982349 & 3.451270 & -0.980237 \\ 1 & 1.154097 & 3.704704 & 0.572961 \\ 1 & 2.373704 & 2.410122 & 0.414660\end{array}$


10 product complex for methanolysis of $\varepsilon-\mathrm{CL}$ with two $\mathrm{CH} 3 \mathrm{OH}$

Total energy $+\mathrm{ZPE}=-616.319062$

$\begin{array}{rrrr}6 & 3.760308 & 0.850283 & 0.925901 \\ 8 & 3.212069 & -0.400618 & 0.470604 \\ 6 & 2.006583 & -0.336585 & -0.119118 \\ 6 & 1.549165 & -1.705469 & -0.585409 \\ 6 & 0.084504 & -1.742644 & -1.032397 \\ 6 & -0.910827 & -1.585412 & 0.125426 \\ 6 & -2.350568 & -1.329729 & -0.340646 \\ 6 & -3.242683 & -0.756786 & 0.769439 \\ 8 & -2.698169 & 0.402981 & 1.374637 \\ 8 & 1.407394 & 0.715865 & -0.265288 \\ 8 & -1.238053 & 1.790164 & -0.659819 \\ 6 & -1.054974 & 3.199106 & -0.674270 \\ 1 & -2.344292 & 0.977982 & 0.663619 \\ 1 & -2.332393 & -0.605959 & -1.167063 \\ 1 & -4.246558 & -0.559566 & 0.353946 \\ 1 & -2.805282 & -2.248443 & -0.737879 \\ 1 & -3.370223 & -1.491457 & 1.575511 \\ 1 & -0.874525 & -2.475604 & 0.770339 \\ 1 & -0.611797 & -0.740201 & 0.754242 \\ 1 & 1.746529 & -2.421183 & 0.221982 \\ 1 & -0.096085 & -2.687098 & -1.561607 \\ 1 & 2.219646 & -1.994079 & -1.407099 \\ 1 & -0.077678 & -0.941631 & -1.764341 \\ 1 & 3.891244 & 1.538708 & 0.087131 \\ 1 & 4.723024 & 0.599564 & 1.371243 \\ 1 & 3.100552 & 1.308867 & 1.666637 \\ 1 & -0.360952 & 1.372050 & -0.554115 \\ 1 & -2.043168 & 3.653703 & -0.785190 \\ 1 & -0.604124 & 3.570815 & 0.257194 \\ 1 & -0.428888 & 3.519817 & -1.519180\end{array}$


TS6 for concerted mechanism for methanolysis of $\varepsilon$-CL with two $\mathrm{CH} 3 \mathrm{OH}$

Total energy $+\mathrm{ZPE}=-616.252737$; transition frequency $=1319 i \mathrm{~cm}^{-1}$

$\begin{array}{rrrr}6 & 2.808710 & -1.082006 & -0.135375 \\ 8 & 1.532632 & -0.687133 & -0.629870 \\ 6 & 0.225873 & -1.260962 & 0.355140 \\ 8 & 0.585278 & -2.009865 & 1.222750 \\ 6 & -0.829358 & -1.590910 & -0.694944 \\ 6 & -1.526247 & -0.457088 & -1.463363 \\ 6 & -2.715123 & 0.184707 & -0.721932 \\ 6 & -2.360197 & 1.124060 & 0.440332 \\ 6 & -1.424713 & 0.525272 & 1.489395 \\ 8 & -0.105770 & 0.308699 & 0.984658 \\ 8 & 1.038775 & 1.651211 & -0.606020 \\ 6 & 2.016378 & 2.558570 & -0.103446 \\ 1 & 0.361177 & 1.165833 & 0.257059 \\ 1 & 1.432733 & 0.532100 & -0.750603 \\ 1 & 1.557198 & 3.543658 & 0.034050 \\ 1 & 2.428806 & 2.224936 & 0.858821 \\ 1 & 2.833391 & 2.655624 & -0.826751 \\ 1 & 3.367636 & -0.219599 & 0.250509 \\ 1 & 3.380867 & -1.549038 & -0.944723 \\ 1 & 2.662916 & -1.800122 & 0.677921 \\ 1 & -0.311019 & -2.253588 & -1.395804 \\ 1 & -1.577546 & -2.208133 & -0.181658 \\ 1 & -1.913320 & -0.891183 & -2.393921 \\ 1 & -0.801634 & 0.307561 & -1.764293 \\ 1 & -3.368111 & -0.618791 & -0.349670 \\ 1 & -3.315724 & 0.750499 & -1.445991 \\ 1 & -3.285247 & 1.441522 & 0.941678 \\ 1 & -1.893852 & 2.038010 & 0.046504 \\ 1 & -1.336308 & 1.200742 & 2.350184 \\ 1 & -1.801223 & -0.433184 & 1.869699\end{array}$


12 reactant complex for methanolysis of $\varepsilon$ - $\mathrm{CL}$ with three $\mathrm{CH} 3 \mathrm{OH}$

Total energy $+\mathrm{ZPE}=--731.995246$

$\begin{array}{rrrr}6 & -3.642515 & 0.773461 & 1.411271 \\ 8 & -2.310988 & 0.388227 & 1.106154 \\ 8 & -2.370871 & -1.457498 & -0.904141 \\ 6 & -2.227938 & -2.803263 & -0.465341 \\ 8 & -0.521315 & 2.213892 & 0.063783 \\ 6 & -1.104729 & 2.772004 & -1.099952 \\ 8 & 0.087346 & -0.609310 & -1.899313 \\ 6 & 0.802080 & -0.286596 & -0.961261 \\ 8 & 0.485159 & -0.756841 & 0.256775 \\ 6 & 1.071323 & -0.218668 & 1.469403 \\ 6 & 2.489919 & -0.720433 & 1.716978 \\ 6 & 3.563815 & -0.017393 & 0.876644 \\ 6 & 3.352526 & -0.106542 & -0.640926 \\ 6 & 2.050215 & 0.550161 & -1.147791 \\ 1 & 2.713852 & -0.570790 & 2.781768 \\ 1 & 2.510552 & -1.804855 & 1.543470 \\ 1 & 3.599327 & 1.043469 & 1.164042 \\ 1 & 4.546567 & -0.437401 & 1.126046 \\ 1 & 4.190884 & 0.395448 & -1.138729 \\ 1 & 3.388249 & -1.155096 & -0.966997 \\ 1 & 1.884583 & 1.514876 & -0.652680 \\ 1 & 2.113272 & 0.741408 & -2.221527 \\ 1 & -2.343663 & -0.316028 & 0.408393 \\ 1 & -4.211408 & -0.053781 & 1.860262 \\ 1 & -3.594304 & 1.589495 & 2.138535 \\ 1 & -4.190331 & 1.128066 & 0.525886 \\ 1 & 1.011407 & 0.872905 & 1.432691 \\ 1 & 0.387948 & -0.567913 & 2.245314 \\ 1 & -2.151879 & -3.494611 & -1.316120 \\ 1 & -1.346996 & -2.934693 & 0.177054 \\ 1 & -3.124103 & -3.060355 & 0.106838 \\ 1 & -1.531264 & -1.203965 & -1.355324 \\ 1 & -1.189601 & 1.613079 & 0.471591 \\ 1 & -0.375215 & 3.458414 & -1.542698 \\ 1 & -1.355174 & 2.008846 & -1.852722 \\ 1 & -2.015522 & 3.349343 & -0.875316\end{array}$


TS7 for addition step of stepwise mechanism for methanolysis of $\varepsilon$-CL with three $\mathrm{CH} 3 \mathrm{OH}$

Total energy $+\mathrm{ZPE}=-731.956901 ;$ transition frequency $=1382 i \mathrm{~cm}^{-1}$

$\begin{array}{rrrr}6 & 0.648562 & 2.502850 & -0.668873 \\ 8 & 0.365683 & 1.480427 & 0.280972 \\ 6 & -0.467490 & 0.045175 & -0.545321 \\ 8 & 0.341437 & -0.369120 & -1.456734 \\ 8 & -1.547130 & 0.652072 & -1.095030 \\ 6 & -2.632170 & 1.073515 & -0.250075 \\ 6 & -3.655126 & -0.044485 & -0.055062 \\ 6 & -3.211776 & -1.165592 & 0.896065 \\ 6 & -1.845617 & -1.793020 & 0.573894 \\ 6 & -0.668988 & -0.804040 & 0.711228 \\ 8 & 2.527891 & 0.618202 & 0.942062 \\ 6 & 2.725091 & 0.378274 & 2.323231 \\ 8 & 2.358182 & -1.302029 & -0.500095 \\ 6 & 3.405266 & -1.438014 & -1.453193 \\ 1 & 1.411199 & 1.072479 & 0.669835 \\ 1 & 2.555240 & -0.404343 & 0.243043 \\ 1 & 1.310955 & -0.889901 & -1.024485 \\ 1 & -0.205560 & 3.183196 & -0.752912 \\ 1 & 0.860138 & 2.070693 & -1.655711 \\ 1 & 1.524894 & 3.069168 & -0.333050 \\ 1 & 3.766934 & 0.086452 & 2.507328 \\ 1 & 2.072767 & -0.419159 & 2.713505 \\ 1 & 2.523021 & 1.293924 & 2.893395 \\ 1 & 4.328316 & -1.744621 & -0.947168 \\ 1 & 3.596492 & -0.499615 & -1.993164 \\ 1 & 3.135762 & -2.211336 & -2.181183 \\ 1 & -0.824519 & -0.160265 & 1.581673 \\ 1 & 0.263410 & -1.350321 & 0.876133 \\ 1 & -1.674291 & -2.623593 & 1.269343 \\ 1 & -1.847743 & -2.228957 & -0.433495 \\ 1 & -3.176741 & -0.770541 & 1.922268 \\ 1 & -3.978688 & -1.950836 & 0.897580 \\ 1 & -4.583967 & 0.403361 & 0.324107 \\ 1 & -3.886516 & -0.456749 & -1.045822 \\ 1 & -3.086034 & 1.909193 & -0.790140 \\ 1 & -2.240092 & 1.457362 & 0.698150\end{array}$


13a tetrahedral adduct intermediate for methanolysis of $\varepsilon$ - $\mathrm{CL}$ with three $\mathrm{CH} 3 \mathrm{OH}$

Total energy $+\mathrm{ZPE}=-731.980828$

$\begin{array}{rrrr}6 & 0.464350 & 2.248844 & -1.276926 \\ 8 & 0.217517 & 1.371147 & -0.170813 \\ 6 & -0.495039 & 0.149829 & -0.521850 \\ 6 & -0.615314 & -0.623741 & 0.805726 \\ 6 & -1.718281 & -1.695484 & 0.802146 \\ 6 & -3.111649 & -1.141366 & 1.146235 \\ 6 & -3.701186 & -0.170492 & 0.113054 \\ 6 & -2.766210 & 0.965886 & -0.303683 \\ 8 & -1.706075 & 0.481122 & -1.131589 \\ 8 & 0.174233 & -0.557557 & -1.497881 \\ 8 & 2.542194 & -1.419369 & -0.359820 \\ 6 & 3.625013 & -1.717474 & -1.231925 \\ 8 & 2.680995 & 0.933089 & 0.985403 \\ 6 & 2.713788 & 1.029969 & 2.403899 \\ 1 & 1.771846 & 1.151833 & 0.670065 \\ 1 & 2.772128 & -0.623491 & 0.180142 \\ 1 & 1.018740 & -0.906604 & -1.116132 \\ 1 & -0.467562 & 2.705235 & -1.624128 \\ 1 & 0.930260 & 1.707679 & -2.105456 \\ 1 & 1.140757 & 3.023429 & -0.907809 \\ 1 & 3.725349 & 0.769745 & 2.727172 \\ 1 & 2.005326 & 0.342215 & 2.887636 \\ 1 & 2.495441 & 2.052583 & 2.740737 \\ 1 & 4.528747 & -1.992158 & -0.670244 \\ 1 & 3.867859 & -0.877109 & -1.897880 \\ 1 & 3.327160 & -2.571875 & -1.845685 \\ 1 & -0.785062 & 0.086127 & 1.624085 \\ 1 & 0.359098 & -1.088083 & 0.990921 \\ 1 & -1.460045 & -2.460470 & 1.544930 \\ 1 & -1.730999 & -2.198567 & -0.172648 \\ 1 & -3.051236 & -0.635525 & 2.121813 \\ 1 & -3.810080 & -1.977706 & 1.280620 \\ 1 & -4.628066 & 0.265955 & 0.511479 \\ 1 & -3.974929 & -0.715439 & -0.799631 \\ 1 & -3.310673 & 1.686401 & -0.923636 \\ 1 & -2.367810 & 1.512538 & 0.561192\end{array}$


13b tetrahedral adduct intermediate for methanolysis of $\varepsilon$ - $\mathrm{CL}$ with three $\mathrm{CH} 3 \mathrm{OH}$

Total energy $+\mathrm{ZPE}=-731.981140$

$\begin{array}{rrrr}6 & 0.713680 & -2.194969 & 1.734391 \\ 8 & -0.523148 & -2.038063 & 1.045860 \\ 6 & -0.513890 & -1.131587 & -0.032312 \\ 6 & -1.821939 & -1.374583 & -0.797360 \\ 6 & -2.353115 & -0.165143 & -1.582181 \\ 6 & -3.186285 & 0.809275 & -0.729371 \\ 6 & -2.403588 & 1.590461 & 0.337430 \\ 6 & -1.512174 & 0.730360 & 1.233319 \\ 8 & -0.394582 & 0.214929 & 0.495219 \\ 8 & 0.540840 & -1.288916 & -0.899450 \\ 8 & 2.958192 & -0.247994 & -0.140376 \\ 6 & 3.871726 & -0.473988 & -1.209060 \\ 8 & 1.530850 & 2.054482 & -0.333889 \\ 6 & 1.601770 & 2.974393 & 0.749598 \\ 1 & 1.391197 & -0.965261 & -0.502664 \\ 1 & -2.059227 & -0.098515 & 1.696364 \\ 1 & -1.088937 & 1.336336 & 2.042715 \\ 1 & -3.107309 & 2.146470 & 0.972836 \\ 1 & -1.762895 & 2.339944 & -0.147966 \\ 1 & -3.987971 & 0.238253 & -0.237772 \\ 1 & -3.687137 & 1.530069 & -1.388844 \\ 1 & -2.993854 & -0.536966 & -2.391507 \\ 1 & -1.517695 & 0.356768 & -2.065612 \\ 1 & -2.579048 & -1.728665 & -0.089156 \\ 1 & -1.610357 & -2.208722 & -1.473089 \\ 1 & 0.498358 & -2.822999 & 2.601887 \\ 1 & 1.115141 & -1.232288 & 2.072174 \\ 1 & 1.460801 & -2.690992 & 1.104419 \\ 1 & 0.752728 & 1.470811 & -0.185653 \\ 1 & 2.493105 & 3.589048 & 0.598130 \\ 1 & 0.726209 & 3.638161 & 0.772626 \\ 1 & 1.686960 & 2.468389 & 1.722012 \\ 1 & 2.633379 & 0.683579 & -0.203089 \\ 1 & 4.767635 & 0.154378 & -1.109539 \\ 1 & 4.178522 & -1.522412 & -1.162605 \\ 1 & 3.416063 & -0.286198 & -2.191293\end{array}$


TS8 for elimination step of stepwise mechanism for methanolysis of $\varepsilon$-CL with three $\mathrm{CH} 3 \mathrm{OH}$

Total energy $+\mathrm{ZPE}=-731.953553$; transition frequency $=1034 i \mathrm{~cm}^{-1}$

$\begin{array}{rrrr}6 & 3.890951 & -0.354280 & 0.656186 \\ 8 & 2.798979 & -0.077430 & -0.215093 \\ 8 & 1.233647 & -1.794784 & -0.869877 \\ 6 & 1.043979 & -2.694448 & 0.235817 \\ 8 & -0.460127 & -0.045665 & -0.792069 \\ 6 & -1.680928 & -0.098112 & -1.518242 \\ 6 & -2.659740 & -1.098788 & -0.901572 \\ 6 & -3.209869 & -0.700462 & 0.476794 \\ 6 & -2.172998 & -0.275834 & 1.534867 \\ 6 & -1.514444 & 1.086432 & 1.264176 \\ 6 & -0.232754 & 1.078187 & 0.419676 \\ 8 & -0.215246 & 2.282303 & -0.284999 \\ 6 & 1.014839 & 2.618807 & -0.914548 \\ 8 & 0.842492 & 0.686408 & 1.020219 \\ 1 & 0.393523 & -1.046748 & -0.862420 \\ 1 & 2.100329 & -1.084392 & -0.618341 \\ 1 & 1.966092 & 0.397785 & 0.336304 \\ 1 & 4.633078 & -0.950925 & 0.115893 \\ 1 & 3.575293 & -0.902601 & 1.554738 \\ 1 & 4.360028 & 0.585203 & 0.969139 \\ 1 & 0.136254 & -3.277778 & 0.060870 \\ 1 & 1.899062 & -3.374112 & 0.282201 \\ 1 & 0.951000 & -2.137888 & 1.174570 \\ 1 & -1.444470 & -0.389374 & -2.551066 \\ 1 & -2.103640 & 0.913741 & -1.557155 \\ 1 & -2.147762 & -2.069626 & -0.831477 \\ 1 & -3.500024 & -1.245893 & -1.595198 \\ 1 & -3.795267 & -1.543000 & 0.869317 \\ 1 & -3.923061 & 0.127479 & 0.348984 \\ 1 & -1.398966 & -1.043691 & 1.657465 \\ 1 & -2.695108 & -0.214859 & 2.498441 \\ 1 & -2.239620 & 1.767569 & 0.805526 \\ 1 & -1.207992 & 1.547549 & 2.209689 \\ 1 & 0.840397 & 3.568450 & -1.425835 \\ 1 & 1.818344 & 2.739441 & -0.179958 \\ 1 & 1.310331 & 1.858009 & -1.646064\end{array}$


14 product complex for methanolysis of $\varepsilon$-CL with three $\mathrm{CH} 3 \mathrm{OH}$

Total energy $+\mathrm{ZPE}=-731.999061$

$\begin{array}{rrrr}6 & -3.796568 & 0.947003 & -1.748217 \\ 8 & -3.555137 & -0.152441 & -0.849756 \\ 6 & -2.484818 & -0.037537 & -0.049475 \\ 6 & -2.354074 & -1.223407 & 0.884112 \\ 6 & -0.923714 & -1.469810 & 1.380750 \\ 6 & 0.036046 & -1.926878 & 0.275059 \\ 6 & 1.480682 & -2.094335 & 0.758392 \\ 6 & 2.460434 & -2.384331 & -0.387842 \\ 8 & 2.429263 & -1.405161 & -1.407793 \\ 8 & -1.754396 & 0.941526 & -0.079705 \\ 8 & 3.017308 & 1.108904 & -0.292615 \\ 6 & 3.123461 & 2.082669 & -1.319610 \\ 8 & 0.691925 & 1.638168 & 1.117347 \\ 6 & 0.457630 & 2.859830 & 1.805196 \\ 1 & 2.712187 & -0.550659 & -1.010802 \\ 1 & 1.797363 & -1.174833 & 1.270832 \\ 1 & 3.476070 & -2.504314 & 0.027280 \\ 1 & 1.546092 & -2.903820 & 1.500907 \\ 1 & 2.197485 & -3.336088 & -0.868475 \\ 1 & -0.322024 & -2.874572 & -0.155028 \\ 1 & 0.039190 & -1.198954 & -0.544716 \\ 1 & -2.763820 & -2.104339 & 0.378130 \\ 1 & -0.965273 & -2.226015 & 2.175565 \\ 1 & -3.025208 & -1.020200 & 1.731633 \\ 1 & -0.538005 & -0.553710 & 1.842351 \\ 1 & -3.963846 & 1.869926 & -1.187158 \\ 1 & -4.688914 & 0.670694 & -2.309611 \\ 1 & -2.945028 & 1.083684 & -2.419049 \\ 1 & 2.206336 & 1.308931 & 0.229001 \\ 1 & 4.005218 & 1.835439 & -1.917347 \\ 1 & 2.247317 & 2.089892 & -1.985315 \\ 1 & 3.258278 & 3.094898 & -0.909782 \\ 1 & -0.135548 & 1.368377 & 0.666574 \\ 1 & 0.184724 & 3.675798 & 1.120829 \\ 1 & -0.334846 & 2.753644 & 2.558518 \\ 1 & 1.386070 & 3.129029 & 2.315642\end{array}$


TS9 for concerted mechanism for methanolysis of $\varepsilon$-CL with three $\mathrm{CH} 3 \mathrm{OH}$

Total energy + ZPE $=-731.934100 ;$ transition frequency $=469 i \mathrm{~cm}^{-1}$

$\begin{array}{rrrr}6 & 0.704065 & -2.566667 & 1.168127 \\ 8 & 0.507774 & -1.169662 & 0.997640 \\ 6 & -0.285972 & -0.823597 & -0.345965 \\ 6 & -1.676711 & -1.478334 & -0.231839 \\ 6 & -2.792870 & -0.713596 & -0.969642 \\ 6 & -3.528102 & 0.315875 & -0.091044 \\ 6 & -2.703915 & 1.534535 & 0.352838 \\ 6 & -1.337619 & 1.198145 & 0.950341 \\ 8 & -0.456149 & 0.712940 & -0.063884 \\ 8 & 0.399404 & -0.979418 & -1.366857 \\ 8 & 1.695081 & 1.807344 & -0.595805 \\ 6 & 1.893216 & 2.985717 & 0.203787 \\ 8 & 2.804670 & -0.125072 & 0.356429 \\ 6 & 3.446952 & -0.942156 & -0.648045 \\ 1 & 0.702273 & 1.406403 & -0.457588 \\ 1 & 2.308951 & 0.927448 & -0.189648 \\ 1 & 1.931104 & -0.588815 & 0.651137 \\ 1 & 1.494965 & -2.710793 & 1.913576 \\ 1 & 1.007241 & -3.044648 & 0.226217 \\ 1 & -0.205966 & -3.058634 & 1.537658 \\ 1 & 3.899733 & -1.812131 & -0.162475 \\ 1 & 2.711813 & -1.251043 & -1.394359 \\ 1 & 4.236140 & -0.339221 & -1.103880 \\ 1 & 1.730572 & 2.781376 & 1.268177 \\ 1 & 1.198475 & 3.757309 & -0.136257 \\ 1 & 2.917922 & 3.330763 & 0.050003 \\ 1 & -1.403979 & 0.463002 & 1.760788 \\ 1 & -0.883435 & 2.104198 & 1.374709 \\ 1 & -2.530162 & 2.198055 & -0.505129 \\ 1 & -3.281799 & 2.113420 & 1.087839 \\ 1 & -3.905187 & -0.204467 & 0.802468 \\ 1 & -4.416177 & 0.676936 & -0.626939 \\ 1 & -3.537646 & -1.438860 & -1.321777 \\ 1 & -2.374304 & -0.238179 & -1.865136 \\ 1 & -1.555746 & -2.471569 & -0.676222 \\ 1 & -1.965301 & -1.629145 & 0.815284\end{array}$

